\title{
Stimulation of wound healing using bioinspired hydrogels with basic fibroblast growth factor (bFGF)
}

This article was published in the following Dove Press journal: International Journal of Nanomedicine

\author{
Xiaoyu Zhang' \\ Xiaoning Kang ${ }^{2}$ \\ Lijun Jin' \\ Jie Bai' \\ Wei Liu' \\ Zunyi Wang
}

'Third Ward of Tumor Surgery Department, Cangzhou Central Hospital, Cangzhou, Hebei Province, People's Republic of China; ${ }^{2}$ Third Ward of Ultrasound Department, Cangzhou Central Hospital, Cangzhou, Hebei Province, People's Republic of China
Correspondence: Zunyi Wang Third Ward of Tumor Surgery Department, Cangzhou Central Hospital, Cangzhou 061000, Hebei Province,

People's Republic of China

Tel +8603I 72075979

Email wangzunyi2018@163.com
Introduction: The objective of this study is to stimulate wound healing using bioinspired hydrogels with basic fibroblast growth factor (bFGF).

Materials and methods: Inspired by the crosslinking mechanism in algae-based adhesives, hydrogels were fabricated with gum arabic, pectin, and $\mathrm{Ca}^{2+}$. The physical properties of the bioinspired hydrogels were characterized, and the in vitro release of bFGF was investigated. Then, the in vitro scratch assay for wound healing and in vivo wound healing experiment in a full-thickness excision wound model were performed for the bioinspired hydrogels with bFGF. Finally, histological examinations and organ toxicity tests were conducted to investigate the wound healing applications of the bioinspired hydrogels with bFGF.

Results: The in vitro and in vivo results showed that the bioinspired hydrogels with bFGF could significantly enhance cell proliferation, wound re-epithelialization, collagen deposition, and contraction without any noticeable toxicity and inflammation compared with the hydrogels without bFGF and commercial wound healing products.

Conclusion: These results suggest the potential application of bioinspired hydrogels with bFGF for wound healing.

Keywords: bioinspired hydrogels, basic fibroblast growth factor, gum arabic, pectin, wound healing

\section{Introduction}

Wound healing is a dynamic and complex process involving biochemical and physiological phenomena such as inflammation, proliferation, and remodeling. ${ }^{1}$ This has raised significant concerns in regard to pathologies including postsurgery wounds, burns, and scars. ${ }^{2}$ The development of new biomaterials could enhance the treatment of wound healing. ${ }^{3-6}$ Traditional wound healing biomaterials normally act as a temporary barrier to stop bleeding and prevent infection. Although many natural and synthetic biomaterials such as protein, gum arabic, and polyurethane have been developed for wound healing applications, most of them are not cost-efficient and not suitable enough for clinical applications. ${ }^{7-12}$ Hydrogels are among the best candidates that can be utilized for wound healing with the moisture keeping and drug-releasing advantages, but until now, most of them are still difficult to be synthesized in a large scale. ${ }^{13-17}$ Therefore, design and fabrication of biocompatible and cost-efficient hydrogels for wound healing applications are still a grand challenge.

Recently, researchers have discovered a naturally occurring adhesive secreted from the brown alga Fucus serratus, which has been demonstrated to stick to both hydrophilic and hydrophobic substrates. ${ }^{18}$ The adhesive is formed in a rigid network 
structure by alginate, phenolic polymer, and calcium. ${ }^{19}$ The calcium ions stimulate crosslinking as an egg-box-like structure. Moreover, the nanostructure has also discovered from the alga-based adhesives. ${ }^{20}$ The highly stretchable and tough hydrogels have been successful synthesized based on this crosslinking mechanism, which has demonstrated the feasibility of the following inspiration. ${ }^{21}$ Therefore, in this study, the synthesis of bioinspired hydrogels was proposed based on the chemical structure and nanostructure of the alga adhesive using pectin, gum arabic, and calcium ions. Pectin is a heterosaccharide found in plant cell walls. ${ }^{22}$ It is very cheap, can be extracted from renewable sources, and has biocompatible properties. It has been investigated for many different biomedical applications, such as drug delivery, tissue engineering, and wound dressing. ${ }^{23}$ Mostly, pectin is utilized in conjunction with other biomaterials such as polymers and collagens due to its poor mechanical properties. ${ }^{24}$ Therefore, pectin is always used as a gelling agent, which is suitable for the formation of hydrogels. ${ }^{25}$ Gum arabic includes arabinose and galactose, which is another polysaccharide that has been extensively used in pharmaceutical, cosmetic, and food industries. ${ }^{26}$ It has been used to cover inflamed surfaces to treat inflammation of the intestinal mucosa, and it also possesses some antibacterial and antioxidant properties, which are additions to the wound healing process. ${ }^{27,28}$ Calcium has an established role in homeostasis of mammalian skin and serves as a modulator of keratinocyte proliferation and differentiation. ${ }^{29}$ Therefore, calcium is one of the major contributors for the bioinspired hydrogels. Recent advances using keratinocytes, dermal fibroblasts, and reconstructed epidermis in culture have identified the capacity of local calcium to modulate cell proliferation, maturation, and creation of epidermal lipid barrier, which plays a vital role in epidermal regeneration and dermal reconstruction in wound healing. ${ }^{30,31}$ All of these features suggest pectin, gum arabic, and calcium ions as potential agents to inspire hydrogels from the alga adhesive for wound healing applications.

In addition to the bioinspired hydrogels, growth factors are also required to enhance the wound healing effects. There are three overlapping stages for the wound healing processes, including inflammation, tissue regeneration, and tissue remodeling. ${ }^{32}$ Researchers have found that many growth factors, such as basic fibroblast growth factor (bFGF), fibroblast growth factor-2, VEGF, epidermal growth factors, and platelet-derived growth factor, are important in different dynamic stages. ${ }^{16,33}$ They can improve cell migration, proliferation, differentiation, and angiogenesis. In this study, the bFGF was selected as a model growth factor to enhance wound healing effects of the bioinspired hydrogels. The $17 \mathrm{kDa}$ polypeptide bFGF is the primary promoter for cell proliferation. ${ }^{34,35}$ It has been demonstrated for the wound healing and bone fracture repair applications. ${ }^{36}$ However, the bFGF could lose its bioactivity rapidly in normal physiological conditions without stabilization. Therefore, it is essential to integrate the bFGF into sustainable drug-releasing systems, such as hydrogels, which can enhance the efficiency of bFGF utilization.

In this study, we investigate whether bioinspired hydrogels combined with bFGF could increase the wound healing process and perform better than the current commercial hydrogel products. With this purpose, the bioinspired hydrogels with bFGF were first synthesized using pectin, gum arabic, and $\mathrm{CaCl}_{2}$. After that, the physical properties of the bioinspired hydrogels were carefully characterized using atomic force microscopy (AFM) and dynamic light scattering (DLS). The in vitro drug release and cell proliferation tests were also performed to investigate the functions and properties of bFGF. In addition, the bioinspired hydrogels with bFGF were assigned for in vitro wound healing tests in scratch assays and in vivo wound healing activity tests in a full-thickness excision wound model. Histological examination and organ toxicity test were also conducted to investigate the wound healing applications of the bioinspired hydrogels with bFGF.

\section{Materials and methods Materials}

Pectin, gum arabic, calcium chloride dihydrate, and bFGF were purchased from Sigma-Aldrich China (Shanghai, People's Republic of China). Fetal calf serum (FCS), streptomycin, and penicillin were purchased from Thermo Fisher Scientific, Waltham, MA, USA. The DMEM cell culture medium was also purchased from Thermo Fisher China. The kit for bFGF detection was purchased from Zhongshan Golden Bridge Biotechnology (Beijing, People's Republic of China). A commercial product for wound healing was purchased from HARTMANN (Heidenheim, Hydrocoll, Germany). All solvents and reagents utilized in this study were of analytical reagent grade.

\section{Preparation of bioinspired hydrogels}

The bioinspired hydrogels were synthesized by the reaction of pectin, gum arabic, and $\mathrm{CaCl}_{2}$. In the beginning, $10 \%$ (w/v) pectin and gum arabic were dissolved in deionized water separately for 30 minutes, followed by 1-hour stirring. Then, the samples were prepared by adding pectin to gum arabic in the ratio 1:1. The final concentration was always kept at 
$10 \%(\mathrm{w} / \mathrm{v})$. After that, $20 \mathrm{mM} \mathrm{Ca}{ }^{2+}$ was introduced to the above samples. Finally, the bFGF was loaded into the bioinspired hydrogels $(1 \mu \mathrm{g} / \mathrm{mL})$ for the following in vitro and in vivo characterizations.

\section{Atomic force microscopy}

To investigate the nanostructures in pectin, gum arabic, and the bioinspired hydrogels, PicoPlus AFM was utilized to scan the samples. A silicon cantilever with alumina reflex coating was utilized in this study. The spring constant of this rectangular shape silicon cantilever was around 12-44 N/m. The results were obtained in the tapping mode at room temperature, which is more suitable for the characterization of these naturally occurring biomaterials and the bioinspired hydrogels.

\section{Dynamic light scattering}

The size distribution of nanoparticles from gum arabic was characterized by DLS at room temperature using Malvern Zetasizer Nano (Malvern, UK). The tests were operated at least three times.

\section{The bFGF release in vitro}

The enzyme-linked immunosorbent assay (ELISA) was utilized to assess the bFGF release kinetics. For this purpose, the bioinspired hydrogels with bFGF were loaded into $100 \mathrm{kDa}$ dialysis membranes first. Then, the samples were immersed in $\mathrm{pH} 7.4 \mathrm{PBS}$ and placed on a shaker at $37^{\circ} \mathrm{C}$. At 1, 2, 3, 4, 6, 8, 10, and 12 hours, and 1, 2, 3, 4, 5, 6, and 7 days, $1 \mathrm{~mL}$ of the PBS solution was obtained and stored at $-20^{\circ} \mathrm{C}$ for the final analysis. Meanwhile, $1 \mathrm{~mL}$ of fresh PBS was added to the system. The release kinetics of bFGF from the bioinspired hydrogels were obtained by ELISA following the manufacturer's protocol. The total 7 days of cumulative release were recorded for the bioinspired hydrogels with bFGF samples.

\section{Cell proliferation}

The purchased L929 dermal fibroblast cells were cultured in DMEM, supplemented with $10 \%$ fetal bovine serum and $1 \%$ penicillin/streptomycin antibiotics. The cells were cultured until around $80 \%$ confluency and maintained at $37^{\circ} \mathrm{C}$ in a $5 \% \mathrm{CO}_{2}$ humidified atmosphere. For the in vitro cell proliferation studies, the bioinspired hydrogels with and without bFGF were placed in a 96-well plate after 1-hour UV light sterilization. Then, 5,000 L929 cells were seeded in the wells. Nontreated groups were used as negative control. The commercial product was utilized as a positive control to study the cell proliferation effects of the bioinspired hydrogels with bFGF. The MTS assays were operated at days 1, 4, and 7 following the manufacturer's protocols.

\section{Scratch wound assay in vitro}

After the confluence, the L929 dermal fibroblast monolayer cell was scratched by a straight line using a sterile pipette tip, which can mimic an incision wound in the literature. ${ }^{37}$ After that, PBS was utilized to wash cells for removing cell debris. Then, the cells were seeded on the top of the control, bioinspired hydrogels with and without bFGF, as well as the commercial product with fresh cell culture medium at $37^{\circ} \mathrm{C}$. The photographs of the scratch wound were recorded at 12 and 24 hours to investigate and analyze the scratch wound assay using different samples. The scratch area was measured using the Image-J software. Digital photographs were obtained using an inverted microscope (Olympus, Japan).

\section{Excision wound model in vivo}

Balb/c mice $(n=24)$ were divided into four groups. The first group was control group without any treatment $(n=6)$. The second group was treated with bioinspired hydrogels only $(n=6)$. The third group was given bioinspired hydrogels with bFGF $(n=6)$. The fourth group was treated with the commercial product as the positive control $(n=6)$. All the animal research studies were performed in compliance with guidelines approved from the Animal Use and Care Administrative Advisory Committee in Cangzhou Central Hospital, Cangzhou, Hebei Province, People's Republic of China. This committee also approved all the experiments in this study. The full-thickness excision wound model was generated in this study for the evaluation of wound healing performance of the bioinspired hydrogels with bFGF. Before the experiment, all mice were anesthetized with isoflurane and the back hair was removed. The wound site was sterilized with $70 \%$ ethanol. A full-thickness skin with $4 \mathrm{~mm}$ diameter was cut by a $4 \mathrm{~mm}$ biopsy punch. The photographs of the wounds were immediately taken using a digital camera. All the mice were housed individually to prevent additional effects on the wound area. For wound closure study, changes in wound area were recorded and photographed on days 3, 7, and 14 . Wounds were removed from the animal after anesthesia for histological and toxicity analysis. Wound closure rate was evaluated using the following equation:

$$
\text { Wound closure }(\%)=\left(\mathrm{A}_{\mathrm{n}} / \mathrm{A}_{0}\right) \times 100
$$

$\left(A_{0}\right.$ : the original wound area; $A_{n}$ : the wound area at the time of days 3, 7, and 11 accordingly). 


\section{Histological analysis}

The removed skin specimens and multiple organs, ie, brain, heart, liver, lung, kidney, and spleen, from each group were collected to evaluate the histological alterations. All of the skin specimens and organs were fixed in 10\% formalin, processed, and embedded in paraffin. The skin specimens were sectioned perpendicular to the wound surface into $4 \mu \mathrm{m}$ sections by standard procedures. Skin sections were stained with H\&E and Masson's trichrome to study the wound closure properties, such as newly formed epithelium, granulation tissue, and collagen deposition. Organ sections were stained with H\&E only for toxicity evaluation.

\section{Statistical analyses}

The data in this research were analyzed as mean \pm SD. The results obtained from the in vitro experiment were operated at least 3 times. Statistical significance was determined using a two-tailed Student's $t$-test. $P<0.05$ was considered to be statistically significant.

\section{Results and discussion}

\section{Preparation and characterization of the bioinspired hydrogels}

The alga polymeric adhesive is composed of alginate and polyphenol that crosslinked by $\mathrm{Ca}^{2+}$, which have been demonstrated for tissue engineering and many other biomedical applications. ${ }^{38-41}$ Inspired by this, the bioinspired hydrogels were synthesized from gum arabic, pectin, and divalent calcium ions (Figure 1). The glucuronic acid at the surface of gum arabic nanoparticles could supply $\mathrm{COO}^{-}$groups. The pectin also contains $\mathrm{COO}^{-}$groups. Crosslinked by the divalent calcium ions, the egg-box-like structures could be formed by the above three components, and finally, the bioinspired hydrogels formed.

AFM is an important tool used to assess the surface morphology and complex three-dimensional (3D) nano- and microstructure of polysaccharides. The AFM images of the gum arabic in Figure 2A reveal its nanostructure with nanoparticles contained. The average size of the nanoparticles in gum arabic obtained from DLS was around $50 \mathrm{~nm}$ (Figure 2B), which was consistent with the AFM result. Figure $2 \mathrm{C}$ shows the nanoparticles composed of nanomorphological structure in the bioinspired hydrogels. The nanoparticles in the bioinspired hydrogels could assemble into a network nanostructure and then formed the hydrogels. It has been reported that the nanoparticle could not only enhance the stiffness of the nanocomposite but also increase the cohesive and adhesive forces of the nanocomposite through extra crosslinking and mechanical interlocking interactions. ${ }^{42-44}$ In other words, the nanoparticles from the gum arabic could be one of the key components to form network nanostructure and then crosslink into hydrogels. To prove this hypothesis, it has been demonstrated that all of the other samples such as individual pectin, divalent cation ions, pectin and divalent cation ions,
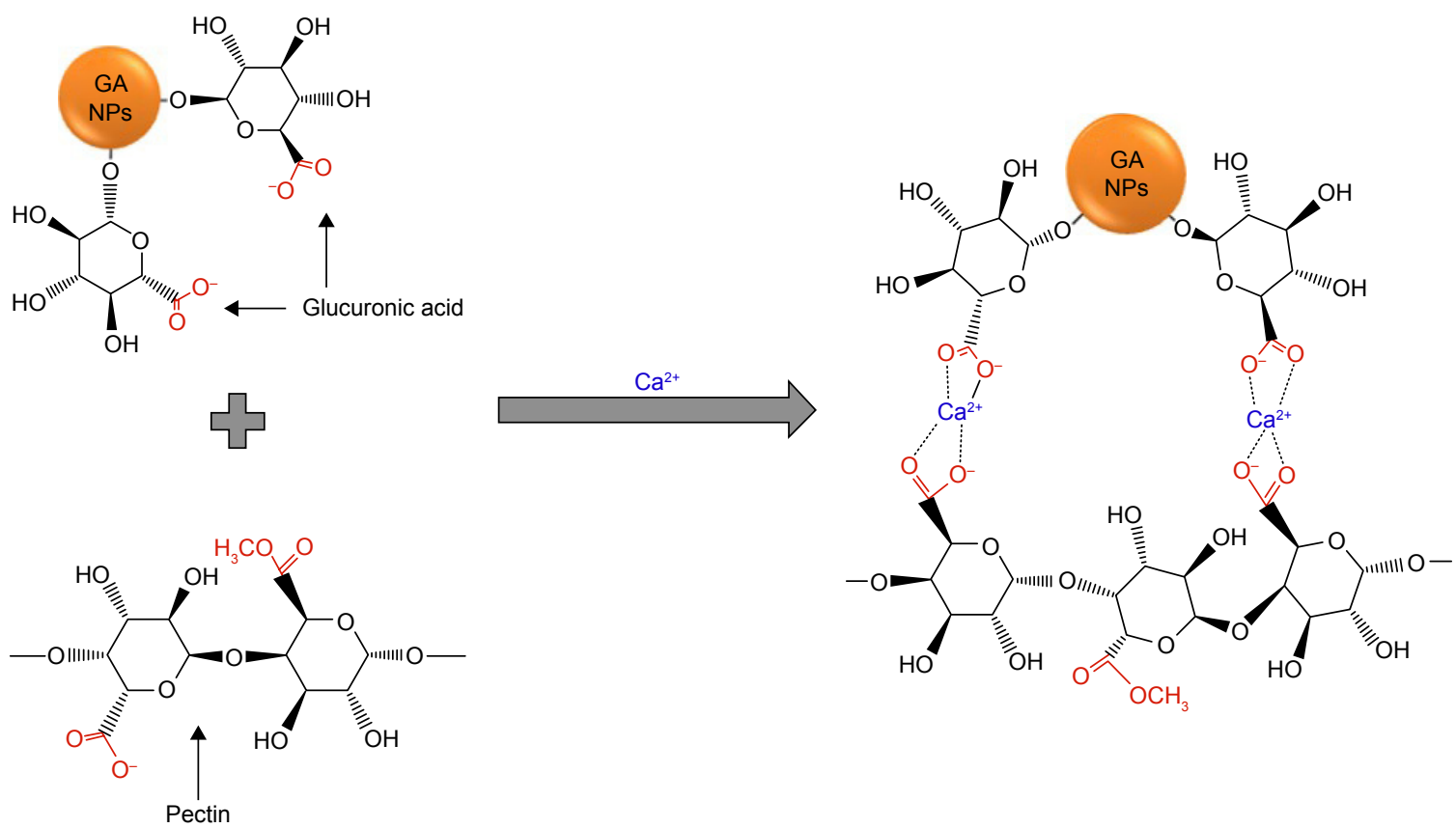

Figure I Chemical structure of the bioinspired hydrogels. 
A

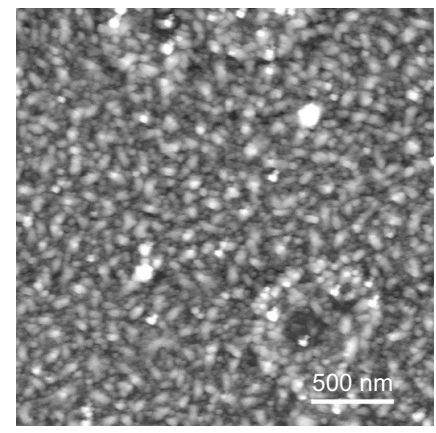

B

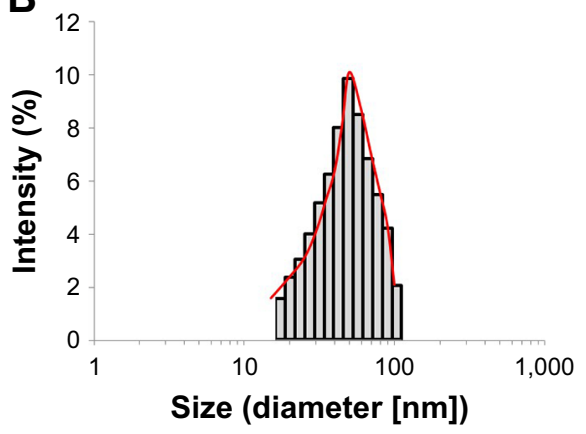

C

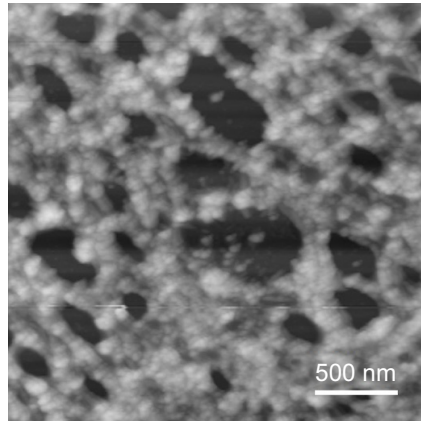

Figure 2 Morphological characterization of the bioinspired hydrogels.

Notes: AFM image (A) and DLS analysis (B) of the nanoparticles from gum arabic, the scale bar=500 nm. The nanostructure (network) investigated from the bioinspired hydrogels (C); the scale bar $=500 \mathrm{~nm}$.

Abbreviations: AFM, atomic force microscopy; DLS, dynamic light scattering.

gum arabic and divalent cation ions, and pectin and gum arabic formed only film structures without apparent network nanostructures. Therefore, they cannot form the hydrogels for wound healing applications. This unique network nanostructure from the bioinspired hydrogels could maintain a physiologically moist microenvironment at the wound site for promoting healing and formation of granulation tissue through the wound healing process. ${ }^{45}$ The potential adhesive forces developed from the bioinspired hydrogels could also supply the adhesion effects at the wound site, which in turn enhanced the wound contraction and closure in the wound healing process. Moreover, this unique nanostructure could serve as an excellent carrier to accelerate the wound healing process. ${ }^{46-48}$

\section{Controlled releasing profile and cell proliferation}

ELISA was utilized to obtain the controlled bFGF release profile from the bioinspired hydrogels (Figure 3). The

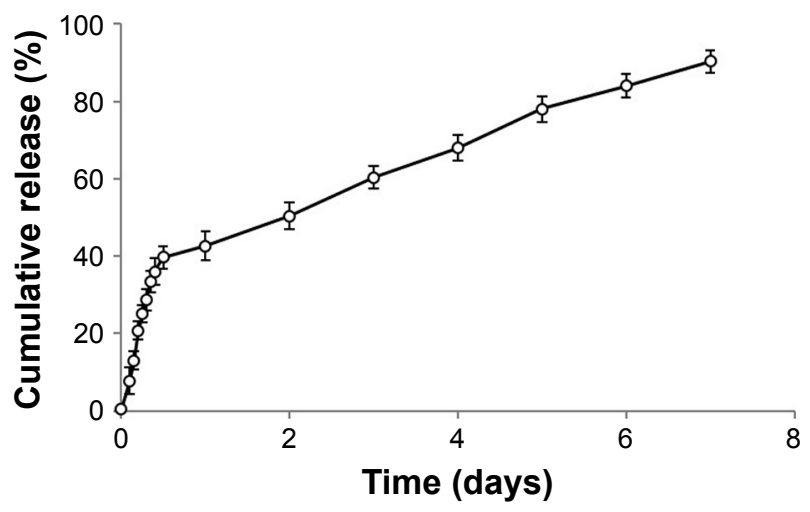

Figure 3 The bFGF release profiles from the bioinspired hydrogels $(n=3)$. Abbreviation: bFGF, basic fibroblast growth factor.
bFGF released from the bioinspired hydrogels displayed an initial burst release around $20 \%$ at 3 hours and around $40 \%$ at 12 hours. After that, a sustained and controlled release profile could be observed for the bFGF release (Figure 3). It is well known that the bFGF can play as one of the major components in fibroblast cell proliferation in the early stage of wound healing process. ${ }^{49}$ Therefore, the controlled burst release of bFGF from the non-crosslinking loosen section of the bioinspired hydrogels in this study could enhance the early-stage wound healing process. Moreover, the bFGF has also shown to be useful in chronic wound healing applications as well as the high-quality scar formation in the later stages of wound healing process. ${ }^{50}$ On the other hand, the bFGF loaded inside the crosslinking section was released slowly could be due to the slow degradation of the egg-boxlike structures formed by the pectin, gum arabic, and $\mathrm{Ca}^{2+}$. So, the controlled and sustained release of bFGF from the bioinspired hydrogels could help the later stages of wound healing processes and have the potential to form scarless wounds finally.

The L929 dermal fibroblast cells were seeded on different samples, including the bioinspired hydrogels with and without bFGF, to test the cell viability and growth through cell proliferation studies (Figure 4). Compared with the control group without any treatment, the bioinspired hydrogels showed increased cell proliferation rates on days 4 and 7 . Moreover, the cell proliferation situation from the bioinspired hydrogels and commercial product was similar on days 1 and 4 . For the bioinspired hydrogels with $\mathrm{bFGF}$, the cell growth and viability were significantly higher than all the other samples, including control, bioinspired hydrogels without bFGF, and the commercial product. The experimental results suggested that both bFGF and bioinspired hydrogels could be the important 


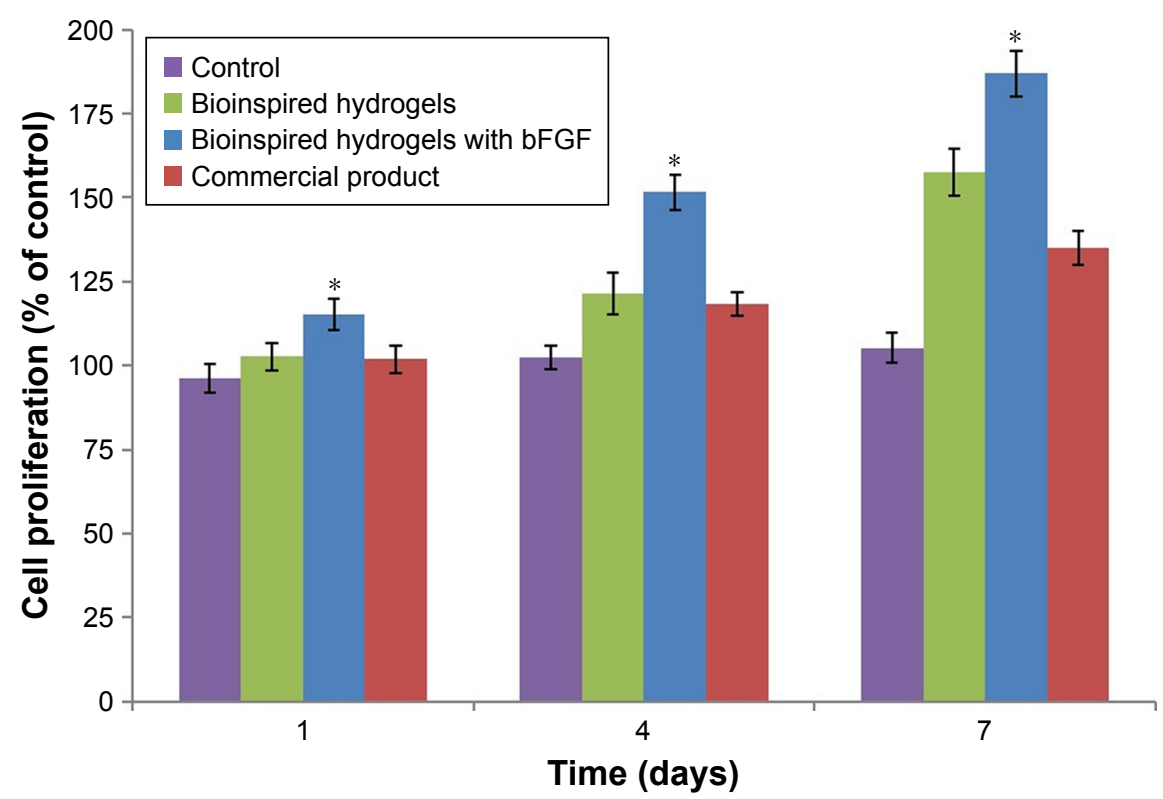

Figure 4 The dermal fibroblast cell proliferation on the bioinspired hydrogels with and without bFGF, negative control (non-treated), and positive control (commercial product; $n=3$ ).

Note: Significant differences between samples means are indicated; $* P<0.05$.

Abbreviation: bFGF, basic fibroblast growth factor.

factors that can affect the dermal fibroblast cell proliferation. More importantly, the bioinspired hydrogels with bFGF system showed the best performance in cell proliferation analysis. As the illustration in the introduction, ${ }^{29,34}$ both $\mathrm{Ca}^{2+}$ and bFGF have the potential to enhance the fibroblast cell proliferation in wound healing processes, which is also compared with the data obtained in this study.

\section{In vitro wound scratch characterizations}

For skin wound healing, cell migration is very important for the wound contraction and the later curing stages. Therefore, studying dermal fibroblast migration affected by different samples may help to target therapies for improved wound healing. The scratch assay method using the L929 dermal fibroblast cell line was used to evaluate the in vitro wound closure effects of the bioinspired hydrogels with bFGF (Figure 5). At 12 hours, the processes of wound closure just started for the control and commercial product groups in fibroblast scratch assays. However, there is obvious wound closure for the bioinspired hydrogels at 12 hours. More importantly, the extent of wound closure of the bioinspired hydrogels with bFGF together for L929 scratch assays has
A

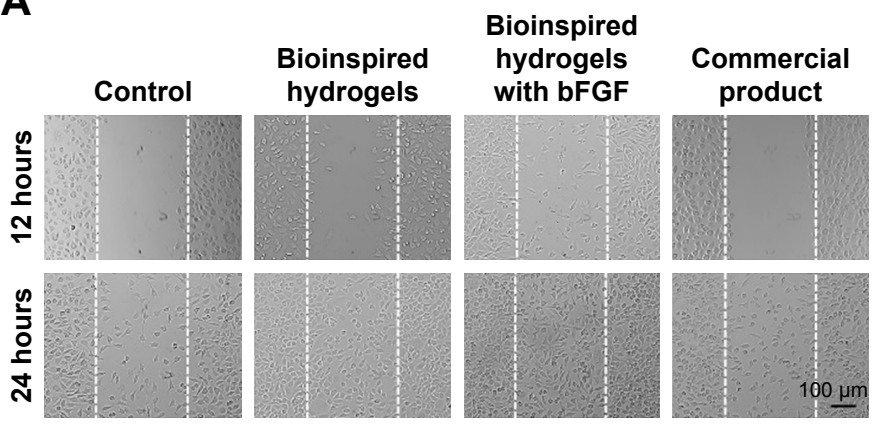

B

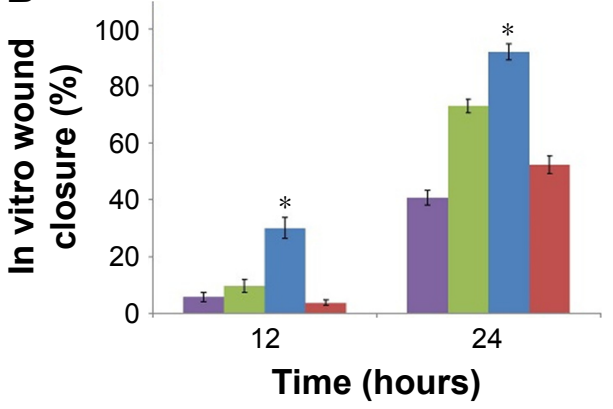

- Control

- Bioinspired hydrogels

- Bioinspired hydrogels with bFGF

- Commercial product

Figure 5 The in vitro scratch assays of L929 dermal fibroblast cells treated with control, bioinspired hydrogels with and without bFGF, and commercial product. Notes: The images $(\mathbf{A})$ and the analyzed results $(\mathbf{B})$ showed the extent of wound closure in scratch assays after 12 and 24 hours for different groups. Error bars indicate SD. Significant differences between samples means are indicated; $* P<0.05$.

Abbreviation: bFGF, basic fibroblast growth factor. 
the highest value. Furthermore, complete wound closure of all the scratches for the bioinspired hydrogels with bFGF together was seen in L929 cultures by 24 hours, compared with all the other samples, including control, bioinspired hydrogels without bFGF, and the commercial product. The bioinspired hydrogels with bFGF enhanced the rate of wound closure significantly at both 12 and 24 hours in L929 fibroblast cell lines. Recent advances using keratinocytes, dermal fibroblasts, and reconstructed epidermis in culture have identified the capacity of local calcium to modulate cell proliferation, maturation, and the creation of epidermal lipid barrier to speed up the wound healing process. These functions that accelerate the wound healing process make the bioinspired hydrogels with bFGF very promising hydrogels for wound healing applications.

\section{In vivo wound closure studies}

To determine the wound closure efficacy of the bioinspired hydrogels with bFGF in vivo, full-thickness skin incisions were created on the back of mice, and then the prepared samples were applied at the wound sites. Figure 6A shows the images of the skin wound taken at different time intervals after treating with control, bioinspired hydrogels with and without bFGF, and the commercial product. There was no sign of inflammation or infection formation in material-covered wounds at different time points. It was observed that the growth of new epidermis was extended to the wound centers in all treated wound lesions, resulting in the reduced area of the wounds. Among the treatment groups, the bioinspired hydrogels with bFGF revealed the most accelerated wound contraction up to 14 days as compared with control, bioinspired hydrogels without bFGF, and the commercial product (Figure 6B). Particularly, the treatment of the bioinspired hydrogels with bFGF led to improved premature healing of the wounds. Three days after transplantation, the wound closure rate of hydrogels with bFGF together reached about $60 \%$, while control, bioinspired hydrogels only, and the commercial product had lower wound closure rates $(27 \%, 42 \%$, and $17 \%$, respectively). However, the bioinspired hydrogels without bFGF still had a valuable wound closure effects compared with the negative and positive controls. Therefore, both of the bioinspired hydrogels and the bFGF could enhance the wound healing in vivo. This result might be due to the material properties of the bioinspired hydrogels, such as nanomorphological structure and $\mathrm{Ca}^{2+}$. These properties could potentially maintain a physiologically moist microenvironment and supply an adhesion effect at the wound site, enhancing the wound contraction and closure at the early stage of the wound healing process. Moreover, it is reported that bFGF could promote the fibroblasts proliferation that
A

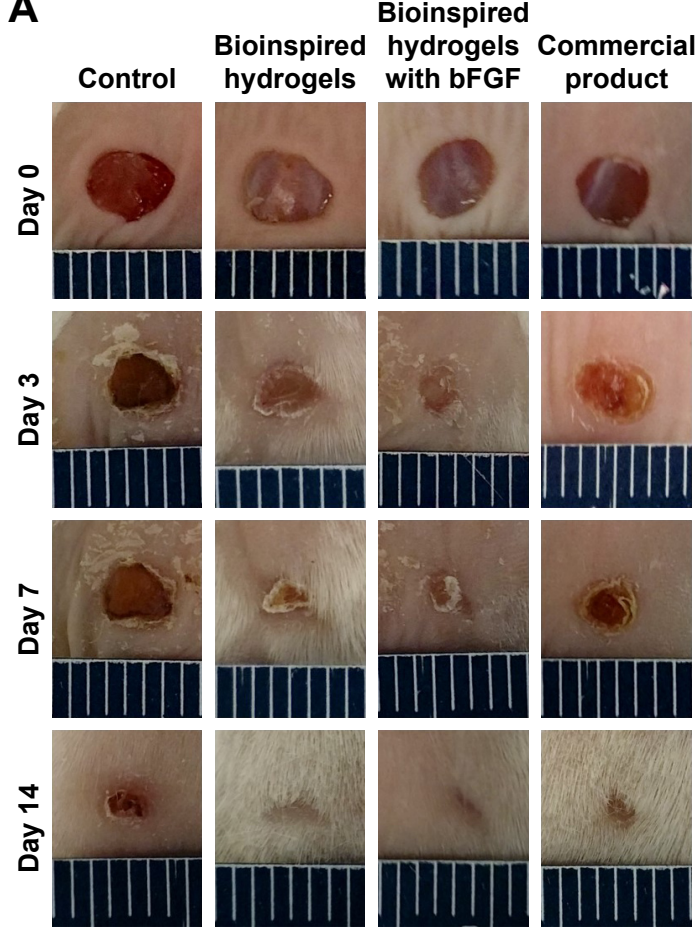

B

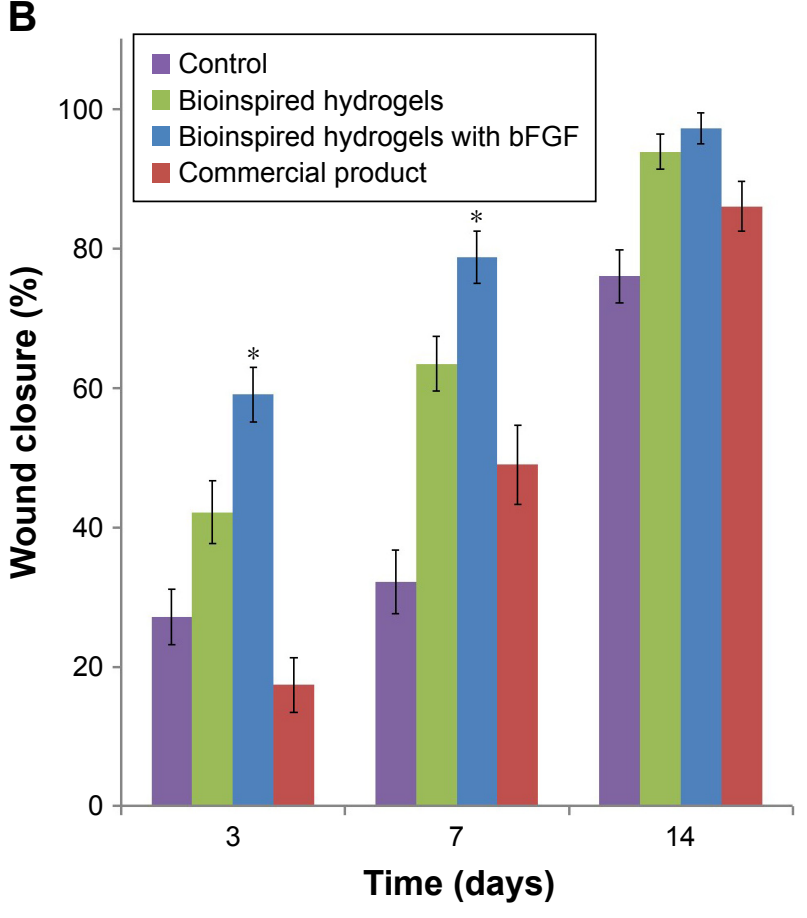

Figure 6 The images (A) and the analyzed results (B) of in vivo wound closure studies for control, bioinspired hydrogels with and without bFGF, and commercial product. Error bars indicate SD.

Note: Significant differences between samples means are indicated; $* P<0.05$.

Abbreviation: bFGF, basic fibroblast growth factor. 
A

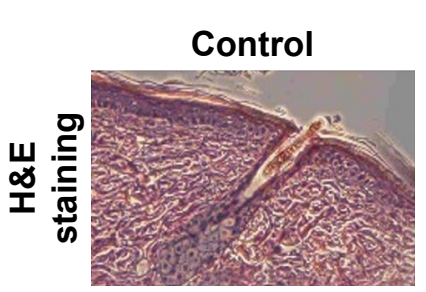

B

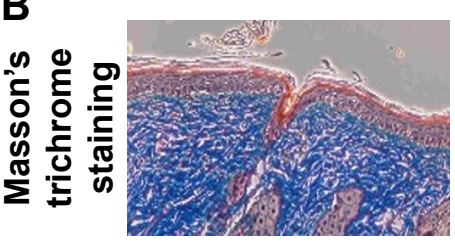

Bioinspired hydrogels with bFGF

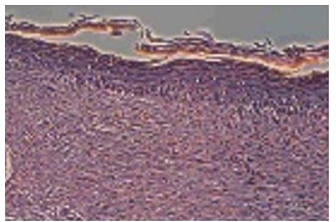

\section{Commercial} product
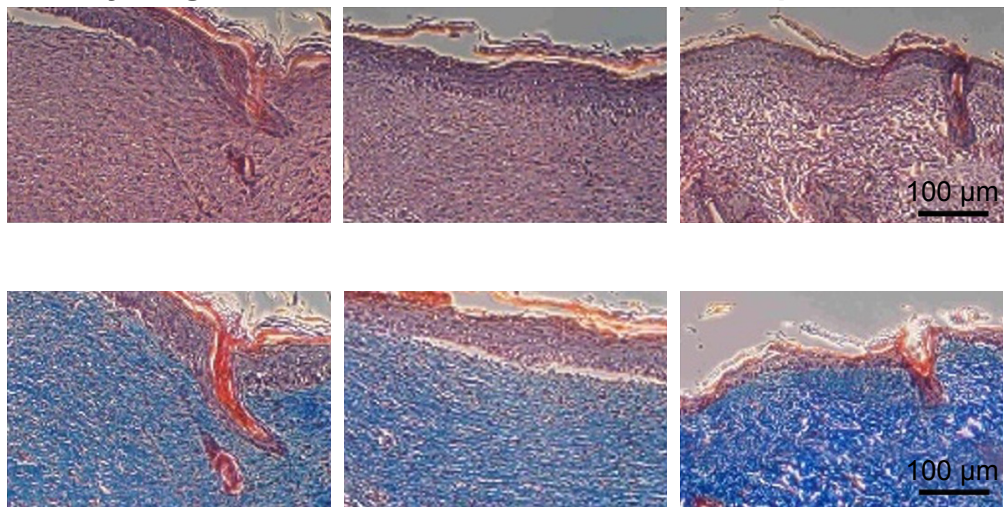

Figure 7 Histological images of H\&E (A) and Masson's trichrome (B) stained sections after 14 days of wound healing. Abbreviation: bFGF, basic fibroblast growth factor.

also could contribute to generating the contractile force at the wound area, and this process mostly occurs at the initial stage of a wound healing process. Both the cell migration that has been demonstrated in the in vitro study and the contractile force generated from the above theory could be the reason of the good performance of the bioinspired hydrogels with bFGF for wound healing applications.

\section{Histological analysis}

Histology of wounds covered with the bioinspired hydrogels with bFGF and other samples on the 14th postoperative day is shown in Figure 7. The surface of the wounds treated with bioinspired hydrogels with bFGF showed a complete formation of new epithelium, which can be observed in Figure 7A stained with H\&E. The fibroblasts' density can also be observed in Figure 7B in which the bioinspired hydrogels with bFGF-treated wound induced the highest fibroblast density. This wound also showed the formation of extracellular proteins with the highest density. The well-proliferated fibroblast and the well-produced collagens in the bioinspired hydrogels with bFGF-treated wound bed contribute to significantly enhance granulation tissue formation. Collagen is a predominant structural protein in skin, which is necessary to effectively reconstruct dermis tissue at the wound sites. After 14 days of treatment, retrieved tissues were processed for Masson's trichrome staining to identify collagen. A more accumulation of the collagen fibers was observed in the wound tissue covered with the bioinspired hydrogels with $\mathrm{bFGF}$, indicating that collagen deposition by the bioinspired hydrogels with bFGF was more mature than the other samples. Furthermore, the bioinspired hydrogels with bFGF-treated wounds showed more densely packed collagen fibers with a parallel arrangement of the collagen fibers in the extracellular matrix. In contrast, loosely packed collagen fibers with irregular arrangement were observed in other samples.

Finally, six major organs, ie, brain, heart, liver, lung, kidney, and spleen, from each experimental animal, were harvested on day 14 and H\&E stained for histological evaluation (Figure 8 ). The local administrated hydrogels could be accumulated in different organs through the blood circulation system, which could also induce some unexpected toxicities. A thorough examination of the H\&E stained organ sections did not reveal any significant signs of toxicity, which further supports the utilization of bioinspired hydrogels with bFGF for wound healing applications.

\section{Conclusion}

This work demonstrated that bioinspired hydrogels based on the chemical structure and nanomorphology of alga adhesive using gum arabic, pectin, and calcium combined with bFGF showed great promise for wound healing applications. The bioinspired hydrogels with unique nanomorphology, promising biocompatibility, controlled growth factor release, and good cell proliferation make them ideal to promote wound healing. The increased fibroblast cells' proliferation indicates that the hydrogels with bFGF could potentially enhance the cell migration for wound healing. The experimental data of in vitro and in vivo wound closure show that the bioinspired hydrogels with bFGF could significantly enhance wound re-epithelialization, collagen deposition, and contraction, which accelerated the wound healing process. The histological organ analysis shows that the bioinspired hydrogels with bFGF did not induce noticeable toxicities. Overall, 


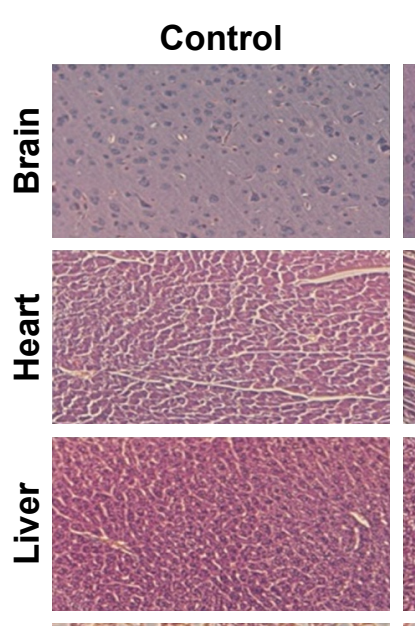

\section{Bioinspired hydrogels}
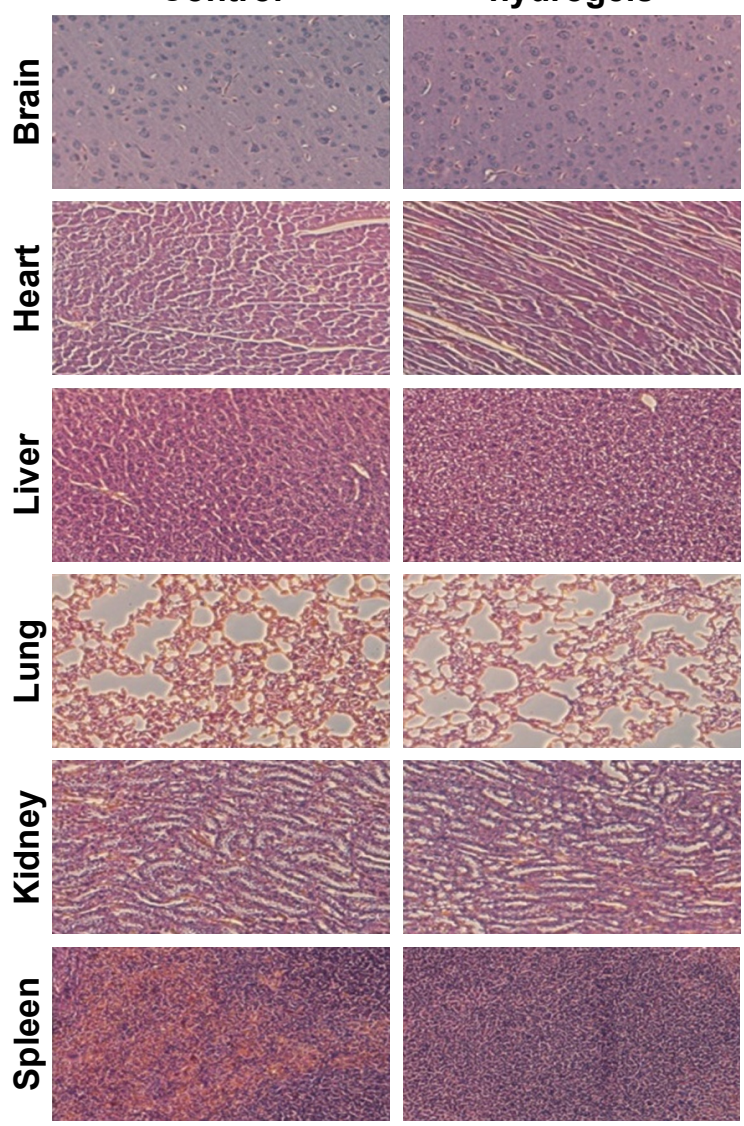

Bioinspired
hydrogels
with bFGF
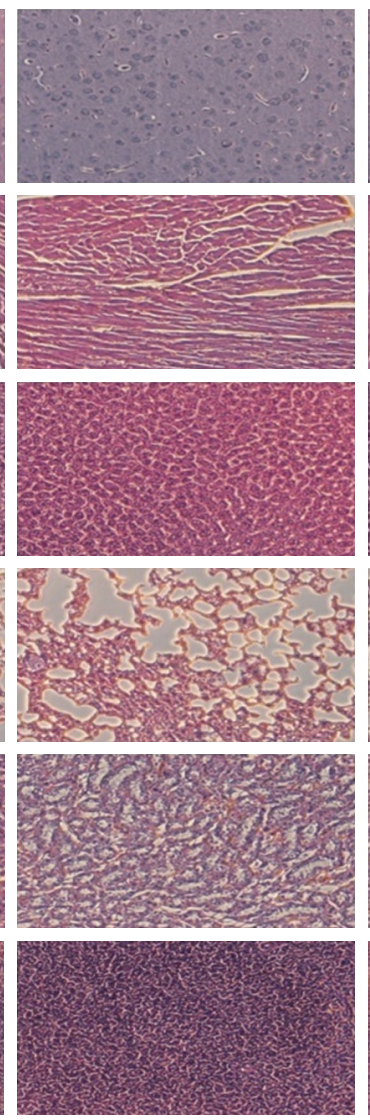

\section{Commercial} product
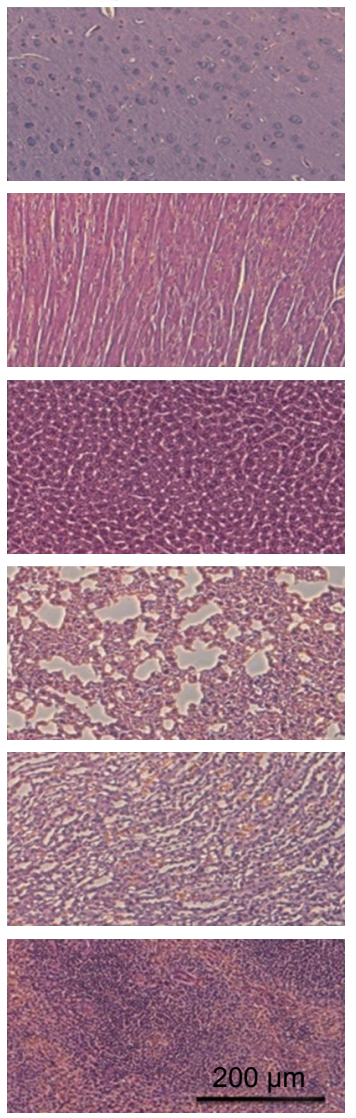

Figure $8 \mathrm{H} \& \mathrm{E}$ stained organs revealed no significant signs of toxicity. Abbreviation: bFGF, basic fibroblast growth factor.

the bioinspired hydrogels with bFGF demonstrate great promise to be utilized for wound healing applications.

\section{Disclosure}

The authors report no conflicts of interest in this work.

\section{References}

1. Guo S, DiPietro LA. Factors affecting wound healing. J Dent Res. 2010; 89(3):219-229.

2. Maalej H, Moalla D, Boisset C, et al. Rhelogical, dermal wound healing and in vitro antioxidant properties of exopolysaccharide hydrogel from Pseudomonas stutzeri AS22. Colloids Surf B Biointerfaces. 2014;123:814-824.

3. Wen Y, Liu W, Bagia C, et al. Antibody-functionalized peptidic membranes for neutralization of allogeneic skin antigen-presenting cells. Acta Biomater. 2014;10(11):4759-4767.

4. Wu J, Wu D, Mutschler MA, Chu CC. Cationic hybrid hydrogels from amino-acid-based poly(ester amide): fabrication, characterization, and biological properties. Adv Funct Mater. 2012;22(18):3815-3823.

5. Wu J, Zhao X, Wu D, Chu CC. Development of a biocompatible and biodegradable hybrid hydrogel platform for sustained release of ionic drugs. J Mater Chem B. 2014;2(38):6660-6668.

6. Gong X. Self-assembly technique for biomedical applications. Nano LIFE. 2015;5(2):1542002.
7. Aramwit P. 1 - Introduction to biomaterials for wound healing A2 - Ågren Magnus S. Wound Healing Biomaterials: Cambridge, UK: Woodhead Publishing; 2016:3-38.

8. Wen Y, Kolonich HR, Kruszewski KM, Giannoukakis N, Gawalt ES, Meng WS. Retaining antibodies in tumors with a self-assembling injectable system. Mol Pharm. 2013;10(3):1035-1044.

9. Mohamed A, Xing M. Nanomaterials and nanotechnology for skin tissue engineering. Int J Burns Trauma. 2012;2(1):29-41.

10. Zhang Z, Gupte MJ, Jin X, Ma PX. Injectable peptide decorated functional nanofibrous hollow microspheres to direct stem cell differentiation and tissue regeneration. Adv Funct Mater. 2015;25(3): 350-360.

11. Li Y, Zhang WB, Hsieh IF, et al. Breaking symmetry toward nonspherical Janus particles based on polyhedral oligomeric silsesquioxanes: molecular design, "click" synthesis, and hierarchical structure. $J$ Am Chem Soc. 2011;133(28):10712-10715.

12. GhavamiNejad A, Rajan Unnithan A, Ramachandra Kurup Sasikala A, et al. Mussel-inspired electrospun nanofibers functionalized with size-controlled silver nanoparticles for wound dressing application. ACS Appl Mater Interfaces. 2015;7(22):12176-12183.

13. Gupta B, Agarwal R, Alam MS. 9 - Hydrogels for wound healing applications A2 - Rimmer, Steve. Biomedical Hydrogels: Cambridge, UK: Woodhead Publishing; 2011:184-227.

14. Elsner JJ, Shefy-Peleg A, Zilberman M. Novel biodegradable composite wound dressings with controlled release of antibiotics: microstructure, mechanical and physical properties. J Biomed Mater Res B Appl Biomater. 2010;93(2):425-435. 
15. Lin J, Li C, Zhao Y, Hu J, Zhang LM. Co-electrospun Nanofibrous membranes of collagen and zein for wound healing. ACS Appl Mater Interfaces. 2012;4(2):1050-1057.

16. Xie Z, Paras CB, Weng H, et al. Dual growth factor releasing multifunctional nanofibers for wound healing. Acta Biomater. 2013;9(12): 9351-9359.

17. Ribeiro MP, Espiga A, Silva D, et al. Development of a new chitosan hydrogel for wound dressing. Wound Repair Regen. 2009;17(6): 817-824.

18. Bitton R, Bianco-Peled H. Novel biomimetic adhesives based on algae glue. Macromol Biosci. 2008;8(5):393-400.

19. Bitton R, Ben-Yehuda M, Davidovich M, et al. Structure of algal-born phenolic polymeric adhesives. Macromol Biosci. 2006;6(9):737-746.

20. Bitton R, Berglin M, Elwing H, et al. The influence of halide-mediated oxidation on algae-born adhesives. Macromol Biosci. 2007;7(12): 1280-1289.

21. Sun JY, Zhao X, Illeperuma WRK, et al. Highly stretchable and tough hydrogels. Nature. 2012;489:133-136.

22. Giusto G, Vercelli C, Comino F, Caramello V, Tursi M, Gandini M. A new, easy-to-make pectin-honey hydrogel enhances wound healing in rats. BMC Complement Altern Med. 2017;17(1):266.

23. Lin HY, Chen HH, Chang SH, Ni TS. Pectin-chitosan-PVA nanofibrous scaffold made by electrospinning and its potential use as a skin tissue scaffold. J Biomater Sci Polym Ed. 2013;24(4):470-484.

24. Munarin F, Tanzi MC, Petrini P. Advances in biomedical applications of pectin gels. Int J Biol Macromol. 2012;51(4):681-689.

25. Ninan N, Muthiah M, Park IK, Elain A, Thomas S, Grohens Y. Pectin/ carboxymethyl cellulose/microfibrillated cellulose composite scaffolds for tissue engineering. Carbohydr Polym. 2013;98(1):877-885.

26. Samy WM, Ghoneim AI, Elgindy NA. Novel microstructured sildenafil dosage forms as wound healing promoters. Expert Opin Drug Deliv. 2014;11(10):1525-1536.

27. Ali BH, Ziada A, Blunden G. Biological effects of gum arabic: a review of some recent research. Food Chem Toxicol. 2009;47(1):1-8.

28. Clark DT, Gazi MI, Cox SW, Eley BM, Tinsley GF. The effects of Acacia arabica gum on the in vitro growth and protease activities of periodontopathic bacteria. J Clin Periodontol. 1993;20(4):238-243.

29. Lansdown AB. Calcium: a potential central regulator in wound healing in the skin. Wound Repair Regen. 2002;10(5):271-285.

30. Lee SH, Elias PM, Proksch E, Menon GK, Mao-Quiang M, Feingold KR. Calcium and potassium are important regulators of barrier homeostasis in murine epidermis. J Clin Invest. 1992;89(2):530-538.

31. Dlugosz AA, Yuspa SH. Protein kinase $\mathrm{C}$ regulates keratinocyte transglutaminase (TGK) gene expression in cultured primary mouse epidermal keratinocytes induced to terminally differentiate by calcium. J Investig Dermatol. 1994;102(4):409-414.

32. Arul V, Kartha R, Jayakumar R. A therapeutic approach for diabetic wound healing using biotinylated GHK incorporated collagen matrices. Life Sci. 2007;80(4):275-284.

33. Barrientos S, Stojadinovic O, Golinko MS, Brem H, Tomic-Canic M. Perspective article: growth factors and cytokines in wound healing. Wound Repair Regen. 2008;16(5):585-601.

34. Edelman ER, Mathiowitz E, Langer R, Klagsbrun M. Controlled and modulated release of basic fibroblast growth factor. Biomaterials. 1991;12(7):619-626.

International Journal of Nanomedicine

\section{Publish your work in this journal}

The International Journal of Nanomedicine is an international, peerreviewed journal focusing on the application of nanotechnology in diagnostics, therapeutics, and drug delivery systems throughout the biomedical field. This journal is indexed on PubMed Central, MedLine, CAS, SciSearch $®$, Current Contents ${ }^{\circledR} /$ Clinical Medicine,
35. Kreuger J, Salmivirta M, Sturiale L, Giménez-Gallego G, Lindahl U. Sequence analysis of heparan sulfate epitopes with graded affinities for fibroblast growth factors 1 and 2. J Biol Chem. 2001;276(33): 30744-30752.

36. Cai S, Liu Y, Zheng Shu X, Prestwich GD. Injectable glycosaminoglycan hydrogels for controlled release of human basic fibroblast growth factor. Biomaterials. 2005;26(30):6054-6067.

37. Salierno MJ, García AJ, del Campo A. Photo-activatable surfaces for cell migration assays. Adv Funct Mater. 2013;23(48):5974-5980.

38. Dimartino S, Lir I, Haber M, Azhari R. Characterization of Biomimetic Adhesives from the Red Alga Gracilaria Conferta for Biomedical Applications. Biological and Biomimetic Adhesives: Challenges and Opportunities: Cambridge, UK: The Royal Society of Chemistry; 2013:117-131.

39. de Jesus Raposo MF, de Morais AM, de Morais RM. Marine polysaccharides from algae with potential biomedical applications. Mar Drugs. 2015;13(5):2967-3028.

40. Noelia F, Maria Jesus GM, Daniela R, Eduarda F, Herminia D, Marisa F. Algae polysaccharides' chemical characterization and their role in the inflammatory process. Curr Med Chem. 2017;24(2):149-175.

41. Tesler AB, Kim P, Kolle S, Howell C, Ahanotu O, Aizenberg J. Extremely durable biofouling-resistant metallic surfaces based on electrodeposited nanoporous tungstite films on steel. Nat Commun. 2015;6:8649.

42. Ahmed MA, Kandil UF, Shaker NO, Hashem AI. The overall effect of reactive rubber nanoparticles and nano clay on the mechanical properties of epoxy resin. J Radiat Res Appl Sci. 2015;8(4):549-561.

43. Raja SN, Olson AC, Limaye A, et al. Influence of three-dimensional nanoparticle branching on the Young's modulus of nanocomposites: effect of interface orientation. Proc Natl Acad Sci US A. 2015;112(21): 6533-6538.

44. ThoniyotP, Tan MJ, Karim AA, Young DJ, Loh XJ. Nanoparticle-hydrogel composites: concept, design, and applications of these promising, multi-functional materials. Adv Sci (Weinh). 2015;2(1-2):1400010.

45. Motta GJ. Calcium alginate topical wound dressings: a new dimension in the cost-effective treatment for exudating dermal wounds and pressure sores. Ostomy Wound Manage. 1989;25:52-56.

46. Wang Y, Yi S, Sun L, Huang Y, Zhang M. Charge-selective fractions of naturally occurring nanoparticles as bioactive nanocarriers for cancer therapy. Acta Biomater. 2014;10(10):4269-4284.

47. Wang Y, Sun L, Yi S, Huang Y, Lenaghan SC, Zhang M. Naturally occurring nanoparticles from arthrobotrys oligospora as a potential immunostimulatory and antitumor agent. Adv Funct Mater. 2013;23(17): 2175-2184.

48. Rice JJ, Martino MM, De Laporte L, Tortelli F, Briquez PS, Hubbell JA. Engineering the regenerative microenvironment with biomaterials. Adv Healthc Mater. 2013;2(1):57-71.

49. Kobayashi F, Matsuzaka K, Inoue T. The effect of basic fibroblast growth factor on regeneration in a surgical wound model of rat submandibular glands. Int J Oral Sci. 2016;8(1):16-23.

50. Akita S, Akino K, Hirano A. Basic fibroblast growth factor in scarless wound healing. Adv Wound Care (New Rochelle). 2013;2(2):44-49.

Journal Citation Reports/Science Edition, EMBase, Scopus and the Elsevier Bibliographic databases. The manuscript management system is completely online and includes a very quick and fair peer-review system, which is all easy to use. Visit http://www.dovepress.com/ testimonials.php to read real quotes from published authors. 